\title{
Clinical sustained uniform ventricular tachycardia in hypertrophic cardiomyopathy: association with left ventricular apical aneurysm
}

\author{
FERNANDO ALFONSO, * MICHAEL P FRENNEAUX, WILLIAM J McKENNA \\ From the Cardiovascular Disease Unit, Hammersmith Hospital, and Department of Cardiological Sciences, St \\ George's Hospital Medical School, London
}

SUMMARY Of 51 patients with hypertrophic cardiomyopathy who had episodes of ventricular tachycardia detected during ambulatory electrocardiographic monitoring only two had clinical ${ }_{-}^{\triangle}$ sustained uniform ventricular tachycardia that required medical treatment because of worsening symptoms. In both patients the arrhythmia was associated with the uncommon finding of an apicalo aneurysm with angiographically normal coronary arteries.

Ventricular tachycardia was detected during ambulatory electrocardiographic monitoring in $20-30 \%$ of unselected adults with hypertrophic cardiomyopathy. ${ }^{12}$ The characteristics of these episodes was relatively homogeneous. ${ }^{3}$ The ventricular tachycardia was slow, non-sustained, and asymptomatic, but it was a marker of subsequent sudden death. ${ }^{1-6}$ Sustained ventricular tachycardia is rare and clinical characterisation of such patients has not been reported. We present two of 51 consecutive patients with ventricular tachycardia in whom episodes were rapid, sustained, and symptomatic; both patients also had a left ventricular apical aneurysm.

\section{Patients and methods}

CASE 1

Hypertrophic cardiomyopathy without a left ventricular gradient was diagnosed in a symptom free $\mathbf{4 0}$ year old man who presented in 1972 with an abnormal electrocardiogram (fig 1a). Angiography showed an apical left ventricular aneurysm with normal coronary arteries (fig 2). Two years later he presented with rapid palpitation and hypotension. Sustained

Requests for reprints to Dr William J McKenna, Department of Cardiological Sciences, St George's Hospital Medical School, Cranmer Terrace, London SW 17 ORE.

^Present address: Fundacion Jimenez Diaz, Madrid, Spain.

Accepted for publication 31 August 1988

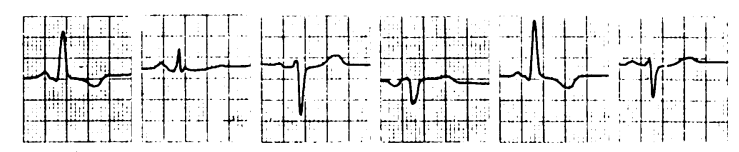

(a) I II III aVR aVL

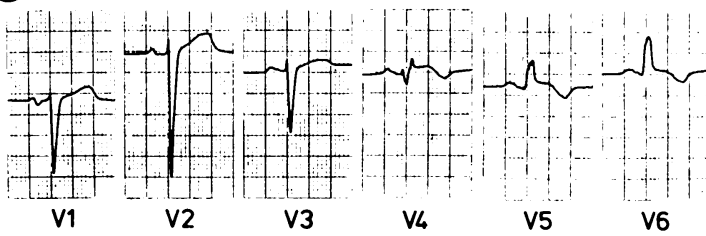

V1

V2

V3

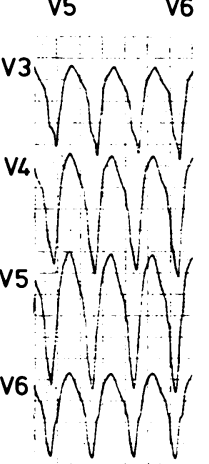

Fig 1 (a) Electrocardiogram from patient 1 showing sinus rhythm with poor $R$ wave progression in the right precordial leads and $S T$ segment elevation in leads V4-V6. (b)

Clinical sustained uniform ventricular tachycardia with right bundle branch block configuration. The axis is $+160^{\circ}$ and the $Q R S$ width $160 \mathrm{~ms}$. A fusion complex (beat 2) is shown in the first panel. 


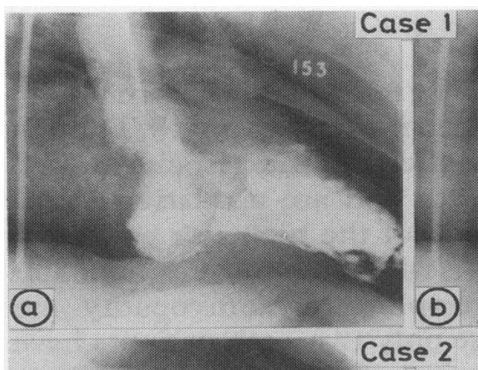

(a) (b)

Fig 2 Diastolic (a) and end systolic frames (b) from left ventricular angiograms (right anterior oblique view) from case 1 (upper) and case 2 (lower). Both patients show systolic mid-cavity elimination with increased residual contrast and paradoxical wall motion at the apex.

uniform ventricular tachycardia ( 180 beats/minute) required DC cardioversion. During a four week stay in hospital repeated episodes of sustained ventricular tachycardia with a consistent configuration (fig 1b) were not controlled with lignocaine, quinidine, or procainamide. An electrophysiological study was performed off medication in 1975. Sustained ventricular tachycardia with the same configuration could be repeatedly induced from the lateral border of the aneurysm and reproducibly terminated by two ventricular extrastimuli. Between 1975 and 1981 drug regimens that included disopyramide ( $2 \mathrm{~g} /$ day) plus propranolol $(120 \mathrm{mg} /$ day $)$ and quinidine Durules (400 mg/day) plus verapamil $(120-240 \mathrm{mg}$ / day) were associated with gastrointestinal and neurological side effects, while dose reductions were associated with recurrence of the arrhythmia. Ventricular arrhythmias did not develop during maximal exercise tests. However, even during asymptomatic periods, episodes of non-sustained ventricular tachycardia were shown during electrocardiographic monitoring. In 1981 all other medications were stopped and amiodarone was started. A daily dose of $400 \mathrm{mg}$ was required to maintain a minimum effective plasma concentration of amiodarone and desethylamiodarone $\left(1.6\right.$ and $1.3 \mathrm{mg} / 1$ respectively). ${ }^{6}$ For the past four years the patient has been symptom free without adverse effects or ventricular arrhythmia during electrocardiographic monitoring. Recent non-invasive evaluation confirmed the diagnosis and the abnormal anatomy. High gain, signal averaged electrocardiography performed by Simson's method ${ }^{7}$ with high-pass filtering at $25 \mathrm{~Hz}$ showed a prolonged filtered QRS duration of $150 \mathrm{~ms}$ (normal $<120 \mathrm{~ms}$ ), a prolonged duration of terminal filtered QRS below $40 \mu \mathrm{V}$ of $41 \mathrm{~ms}$ (normal $<40 \mathrm{~ms}$ ), and a reduced root mean square voltage in the last $40 \mathrm{~ms}$ of $18 \cdot 1 \mu \mathrm{V}$ (normal $>25 \mu \mathrm{V}$ ). Cross sectional echocardiography showed asymmetric septal hypertrophy with maximum wall thickness of $2.2 \mathrm{~cm}$ in the septum and left ventricular free wall at papillary muscle level. There was hyperdynamic contraction at the base of the left ventricle with mid-cavity elimination and an apical aneurysm which was best visualised from the apical two chamber view. There were no echocardiographic or Doppler features of a gradient but colour Doppler showed evidence of mild mitral regurgitation. Phase analysis of technetium-99m radionuclide ventriculography showed that the apex was $180^{\circ}$ out of phase with the body of the left ventricle.

\section{CASE 2}

A 56 year old Indian man with familial hypertrophic cardiomyopathy and a history of exertional chest pain and syncope was referred after a syncopal episode associated with palpitation during which sustained uniform ventricular tachycardia was documented. The tachycardia was at a rate of 190 beats/minute with right bundle branch block configuration, an axis of $-120^{\circ}$, and a QRS width of 140 ms. Over a 40 year period he had experienced six other syncopal episodes that were not associated with chest pain or palpitation. Episodes of exertional chest pain began when he was 26 , were often prolonged (1-2 hours), but were not associated with characteristic electrocardiographic changes of ischaemia or with increased plasma concentrations of myocardial enzymes. They were not consistently relieved by nitrates but treatment with verapamil was associated with fewer episodes (about one a month) than $\beta$ blockers. An electrophysiological study was performed off medication in India. Single and double ventricular extrastimuli failed to induce sustained ventricular arrhythmias. Treatment with sotalol, verapamil, and verapamil plus procainamide failed to suppress episodes of non-sustained ventricular tachycardia during electrocardiographic monitoring.

Our investigations, which were performed after antiarrhythmic drugs were stopped, showed an abnormal electrocardiogram (fig $3 a$ ) and frequent ventricular extrasystoles ( $>1000$ daily) with daily episodes (1-5, median 3) of uniform non-sustained ventricular tachycardia at a rate of $160-180$ beats/ minute (fig $3 b$ ). Exercise testing was limited by fatigue and breathlessness at a maximal oxygen consumption of $26 \mathrm{ml} / \mathrm{kg}$ per minute and no arrhythmia or additional ST segment changes were seen. Cross sectional echocardiography showed asymmetric septal hypertrophy and a small left ventricular apical chamber that expanded during systole. The 


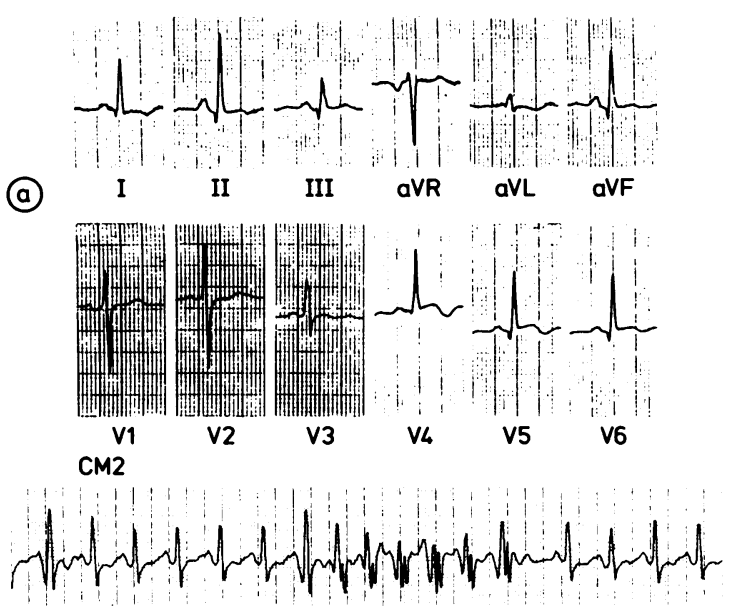

(b) $\mathrm{CM5}$

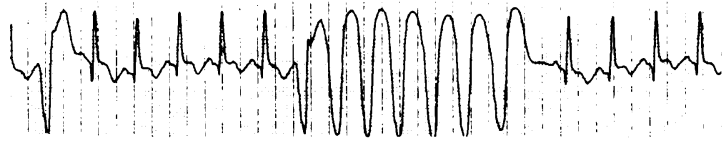

Fig 3 (a) Electrocardiogram from case 2 showing sinus rhythm with a normal $Q R S$ axis and non-pathological $Q$ waves in inferolateral leads. The pattern of $S T$ segment abnormality and elevation in precordial leads V4-V6 is similar to that seen in case 1. (b) Continuous ambulatory electrocardiographic recording of an episode of non-sustained ventricular tachycardia that was similar in configuration to the sustained episode and to other non-sustained episodes.

wall was thickest $(2.4 \mathrm{~cm})$ in the anterior and posterior septum and the free wall at the level of the tip of the mitral valve; there was incomplete systolic anterior motion of the mitral valve. The papillary muscles were greatly hypertrophied with systolic mid-cavity elimination that was associated with a mosaic pattern of turbulent flow on colour Doppler. At cardiac catheterisation there was no resting left ventricular outflow tract or mid-cavity gradient and the left ventricular end diastolic pressure was 26 $\mathrm{mm} \mathrm{Hg}$. Left ventricular angiography (fig 2) showed an akinetic area at the apex with dynamic contraction at the base; the coronary arteries were angiographically normal, apart from a separate origin of the left circumflex and systolic compression of septal perforator arteries. Radionuclide ventriculography with technetium-99m showed mid-cavity elimination with an apical aneurysm that on phase analysis was $180^{\circ}$ out of phase with the remainder of the left ventricle. During the past year the patient was symptom free on amiodarone $400 \mathrm{mg}$ daily with plasma concentrations of amiodarone and desethylamiodarone of 1.4 and $1.1 \mathrm{mg} / 1$ respectively. During 24 hour electrocardiographic monitoring there was a maximum of 240 uniform ventricular extrasystoles and no ventricular tachycardia.

\section{Discussion}

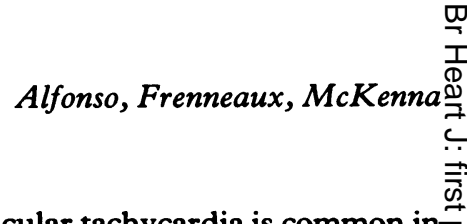

Non-sustained ventricular tachycardia is common inz adults with hypertrophic cardiomyopathy. ${ }^{12}$ Though $\overline{0}$ episodes are asymptomatic they are associated with $\frac{\bar{\omega}}{1}$ subsequent sudden death and are a relatively sen- $\overparen{\otimes}$ sitive and specific marker of the high risk patient. ${ }^{2-6} \stackrel{0}{0}$ Clinical episodes of sustained uniform ventricular ${ }^{\text {s }}$ tachycardia in hypertrophic cardiomyopathy are. rare. To our knowledge only two such patients have $\vec{\omega}$ been reported. ${ }^{8}$ In a review of published reports, six $\stackrel{\omega}{\circ}$ other patients were selected for electrophysiological study because of clinical ventricular tachycardia oros ventricular fibrillation, but no haemodynamic, $\overrightarrow{\text { N }}$ echocardiographic, or additional arrhythmia data $-\overrightarrow{-}$ were available. ${ }^{910} \mathrm{~A}$ patient with polymorphic ven- $\infty$ tricular tachycardia who died suddenly after a period응 of autonomic disturbance and ischaemia has recently been reported. ${ }^{11}$

Both of our patients with sustained uniform ven- $\frac{\mathbb{O}}{2}$ tricular tachycardia also had a left ventricular@ aneurysm with angiographically normal coronary arteries. The apical aneurysms were shown angio- $\overrightarrow{0}$ graphically and confirmed by echocardiographic and radionuclide studies. We did not see this configuration in the 49 patients with non-sustained ventricular tachycardia or in over 200 patients with hypertrophic cardiomyopathy without ventricular tachycardia who were similarly evaluated. Apical aneurysm in $\frac{\varnothing}{\varnothing}$ hypertrophic cardiomyopathy has been reported but $\vec{F}$ is rare and is usually associated with normal coronary윽 arteries. ${ }^{12-14} \mathrm{~A}$ recent report suggested that the $\frac{J}{\sigma}$ presence of mid-ventricular obstruction may predispose to wall motion abnormalities in the distal left ventricle. ${ }^{13}$ When our patients were first examined $\widetilde{\Phi}$ there was no evidence of a left ventricular gradient; however, mid-ventricular obstruction earlier in their course cannot be excluded. Alternatively the apical myocardial changes may have been caused by coron- $O$ ary artery spasm with myocardial infarction. ${ }^{14}$

The electrocardiograms in our patients showed 9 diagnostic clues with precordial ST segment eleva- $\rightarrow$ tion in the absence of abnormal $Q$ waves. This pattern was reported by Gordon et al in three patients $N_{\mathcal{O}}$ with apical aneurysm. ${ }^{15}$ Similar electrocardiographic changes were not seen in a review of electrocardiograms from 100 consecutive patients with hyper- $\omega$ trophic cardiomyopathy who did not have left ven $-\mathcal{F}$ tricular aneurysm..$^{16} \mathrm{We}$ agree with Gordon's suggestion that these electrocardiographic features may be $a_{\infty}^{\Phi}$ marker of ventricular aneurysm. ${ }^{15}$ The patient in whom high gain, signal averaged electrocardio- 0 graphy was performed (case 1) had late potentials. $\overrightarrow{\mathbb{N}}$ The association between the presence of late poten- $\frac{?}{\square}$ tials and a propensity to ventricular tachycardia, $\propto$ especially in patients with left ventricular aneurysm, has been well established, ${ }^{17}$ though the relevance of $\delta$ such a finding in patients with hypertrophic cardio- 
myopathy has not yet been systematically investigated.

Sustained uniform ventricular tachycardia is also uncommon during programmed electrical stimulation studies for the evaluation of clinical arrhythmias or identification of high risk patients with hypertrophic cardiomyopathy. $.^{8-10} 18-24$ More commonly multiform ventricular tachycardia or ventricular fibrillation or both is induced. The clinical significance of these induced arrhythmias is controver-

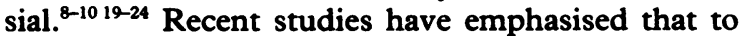
enhance the specificity of the results less vigorous protocols should be used..$^{2024}$

The prognostic significance of clinical sustained uniform ventricular tachycardia in hypertrophic cardiomyopathy, whether or not it is related to the presence of apical aneurysm, remains to be established. Both patients are symptom free and without recurrence of arrhythmia during long term treatment with amiodarone. Should it be necessary, surgical aneurysmectomy remains an option.

\section{References}

1 Savage DD, Seides SF, Maron BJ, Myers DM, Epstein SE. Prevalence of arrhythmia during 24 hour electrocardiographic monitoring and exercise testing in patients with obstructive and non-obstructive hypertrophic cardiomyopathy. Circulation 1979; 59:866-75.

2 McKenna WJ, England D, Doi YL, Deanfield JE, Oakley CM, Goodwin JF. Arrhythmia in hypertrophic cardiomyopathy: I. Influence on prognosis. Br Heart J 1981;46:168-72.

3 McKenna WJ, Krikler DM, Goodwin JF. Arrhythmias in dilated and hypertrophic cardiomyopathy. Med Clin North Am 1984;68:983-1000.

4 Maron BJ, Savage DD, Wolfson JK, Epstein SE. Prognostic significance of 24 hour ambulatory electrocardiographic monitoring in patients with hypertrophic cardiomyopathy. A prospective study. Am J Cardiol 1981;48:252-7.

5 McKenna WJ. Sudden death in hypertrophic cardiomyopathy: Identification of the "high risk" patient. In: Brugada P, Wellens HJJ, eds. Cardiac arrhythmias: where to go from here? Mount Kisco, New York: Futura, 1987:353-65.

6 McKenna WJ, Harris L, Rowland E, et al. Amiodarone for long-term management of hypertrophic cardiomyopathy. Am J Cardiol 1984;54:802-10.

7 Simson MB. Use of signals in the terminal QRS complex to identify patients with ventricular tachycardia after myocardial infarction. Circulation 1981;64:235-42.

8 Geibel A, Brugada P, Zehender M, Stevenson $W$, Waldecker B, Wellens HJJ. Value of programmed electrical stimulation using a standardized ventricular stimulation protocol in hypertrophic cardiomyopathy. Am J Cardiol 1987;60:738-9.

9 Borggrefe M, Podczeck A, Breithardt G. Electrophysiologic studies in hypertrophic cardiomyopathy [Abstract]. Circulation 1986;74(suppl II):II-1922.
10 Kowey PR, Eisenberg R, Engel TR. Sustained arrhythmias in hypertrophic obstructive cardiomyopathy. $N$ Engl J Med 1984;310:1566-9.

11 Nicod P, Polikar R, Peterson KL. Hypertrophic cardiomyopathy and sudden death. $N$ Engl $J$ Med 1988;318:1255-7.

12 Barbaresi F, Longhini C, Brunazzi C, et al. Idiopathic apical left ventricular aneurysm in hypertrophic cardiomyopathy. Jpn Heart J 1985;26:481-94.

13 Fighali S, Krajcer Z, Edelman S, Leachman RD. Progression of hypertrophic cardiomyopathy into a hypokinetic left ventricle: higher incidence in patients with midventricular obstruction. J Am Coll Cardiol 1987;9:288-94.

14 Maron BJ, Epstein SE, Roberts WC. Hypertrophic cardiomyopathy and transmural myocardial infarction without significant atherosclerosis of the extramural coronary arteries. Am J Cardiol 1979;43: 1086-102.

15 Gordon EP, Henderson MA, Rakowski H, Wigle ED. Midventricular obstruction with apical infarction and aneurysm formation [Abstract]. Circulation 1984; 70(suppl II):II-145.

16 McKenna WJ, Borggrefe M, England D, Deanfield J, Oakley CM, Goodwin JF. The natural history of left ventricular hypertrophy in hypertrophic cardiomyopathy: an electrocardiographic study. Circulation 1982;66:1233-40.

17 Breithardt G, Becker R, Seipel L, Abendroth R-R, Ostermeyer J. Non-invasive detection of late potentials in man-a new marker for ventricular tachycardia. Eur Heart J 1981;2:1-11.

18 James TN, Marshall TK. De subitaneis mortibus XII. Asymmetrical hypertrophy of the heart. Circulation 1975;51:1149-66.

19 Watson RM, Liberati Schwartz JM, Maron BJ, Tucker E, Rosing DR, Josephson ME. Inducible polymorphic ventricular tachycardia and ventricular fibrillation in a subgroup of patients with hypertrophic cardiomyopathy at high risk for sudden death. $\mathrm{J} \mathrm{Am}$ Coll Cardiol 1987;10:761-74.

20 Kuck K-H, Kunze K-P, Schluter M, Nienaber CA, Costard A. Programmed electrical stimulation in hypertrophic cardiomyopathy. Results in patients with and without cardiac arrest or syncope. Eur Heart $J$ 1988;9:177-85.

21 Schiavone WA, Maloney JD, Lever HM, Castle LW, Sterba R, Morant V. Electrophysiologic studies of patients with hypertrophic cardiomyopathy presenting with syncope of undetermined etiology. $P A C E$ 1986;9:476-81.

22 Anderson KP, Stinson EB, Derby GC, Oyer PE, Mason JW. Vulnerability of patients with hypertrophic obstructive cardiomyopathy to ventricular arrhythmia induction in the operating room. $\mathrm{Am} \mathrm{J}$ Cardiol 1983;51:811-6.

23 Ingham RE, Mason JW, Rossen RM, Goodman DJ, Harrison DC. Electrophysiologic findings in patients with idiopathic hypertrophic subaortic stenosis. $\mathrm{Am} \mathrm{J}$ Cardiol 1978;41:811-6.

24 Kunze K-P, Kuck K-H, Geiger M, Bleifeld W. Programmed electrical stimulation in hypertrophic cardiomyopathy-specificity and sensitivity of different stimulation protocols [Abstract]. J Am Coll Cardiol 1986;7:195A. 